\title{
Fruit and Vegetable Intake, Physical Activity, and Mortality in Older Community-Dwelling Women
}

\author{
Emily J. Nicklett, PhD, MSW, Richard D. Semba, MD, MPH, Qian-Li Xue, PhD, ${ }^{\dagger}{ }^{\dagger}$ Jing Tian, MS, ${ }^{\S}$ \\ Kai Sun, PhD, MS, Anne R. Cappola, MD, ScM, Eleanor M. Simonsick, PhD, ${ }^{+* * *}$ \\ Luigi Ferrucci, MD, PhD,,$^{+^{* *}}$ and Linda P. Fried, MD, MP $H^{\neq \neq \mathcal{S}}$
}

OBJECTIVES: To examine the relationship between fruit and vegetable intake, physical activity, and all-cause mortality in older women.

DESIGN: Six Cox proportional hazards models examined independent and additive relationships between physical activity, carotenoids, and all-cause mortality. Additional models tested whether physical activity and carotenoids were conjointly related to mortality. Models were adjusted for age, education, and race and ethnicity.

SETTING: Baltimore, Maryland.

PARTICIPANTS: Seven hundred thirteen women aged 70 to 79 participating in the Women's Health and Aging Studies.

MEASUREMENTS: Total serum carotenoids, a marker of fruit and vegetable intake, and physical activity were measured at baseline. Physical activity was measured according to kilocalorie expenditure.

RESULTS: During 5 years of follow-up, $82(11.5 \%)$ participants died. Measured continuously, physical activity improved survival $\quad(\mathrm{HR}=0.52,95 \% \quad \mathrm{CI}=0.41-0.66$, $P<.001)$. The most active women were more likely to survive than the least physically active women $(\mathrm{HR}=0.28,95 \% \mathrm{CI}=0.13-0.59, P<.001)$. Continuous measures of carotenoids improved survival $(\mathrm{HR}=0.67$, $95 \% \mathrm{CI}=0.51-0.89, P=.01)$. Women in the highest tertile of total carotenoids were more likely to survive those in the lowest $(\mathrm{HR}=0.50,95 \% \mathrm{CI}=0.27-0.91, P=.03$ ). When examined in the same model, continuous measures

From the "School of Social Work, University of Michigan, Ann Arbor, Michigan; ${ }^{\dagger}$ Department of Medicine, and ${ }^{\star}$ Division of Geriatric Medicine and Gerontology, and ${ }^{\S}$ Department of Biostatistics, "Division of Endocrinology, Diabetes, and Metabolism, Perelman School of Medicine, University of Pennsylvania, Philadelphia, Pennsylvania; " Intramural Research Program, ${ }^{\dagger}$ Longitudinal Studies Section, National Institute on Aging, Bethesda, Maryland; ${ }^{*}$ Mailman School of Public Health,

${ }^{\S}$ Division of Epidemiology, Department of Medicine, College of

Physicians and Surgeons, Columbia University, New York, New York.

Address corresponding to Emily J. Nicklett, University of Michigan School of Social Work, 1080 S. University Avenue, Rm. 2773, Ann Arbor, MI 48109. E-mail: enicklet@umich.edu

DOI: 10.1111/j.1532-5415.2012.03924.x of physical activity $(\mathrm{HR}=0.54,95 \% \mathrm{CI}=0.42-0.68$, $P<.001)$ and carotenoids $(\mathrm{HR}=0.76,95 \% \mathrm{CI}=0.59$ $0.98, P=.04)$ predicted survival during follow-up.

CONCLUSION: The combination of low total serum carotenoids and low physical activity, both modifiable risk factors, strongly predicted earlier mortality. These findings provide preliminary support that higher fruit and vegetable intake and exercise improve survival. J Am Geriatr Soc 60:862-868, 2012.

Key words: diet; carotenoids; physical activity; mortality; women

$I^{n}$ ndividuals aged 65 and older are now the fasting-growing segment of the population. ${ }^{1,2}$ In addition, chronic, noncommunicable diseases attributable to modifiable risk factors such as tobacco use, diet, and exercise are the leading causes of death in the United States. ${ }^{3,4}$ Given the recent success in smoking cessation, it is likely that maintenance of a healthy diet and a high level of physical activity will become the strongest predictors of health and longevity, and all three are major factors globally.

Fruit and vegetable intake as indicated by total serum carotenoids $^{5-7}$ and physical activity ${ }^{3,8,9}$ have been identified as strong predictors of survival in older adults. Carotenoids are major dietary antioxidants and have been shown to be protective of overall health ${ }^{10}$ and against negative health events such as coronary heart disease, ${ }^{11}$ diseasespecific mortality, ${ }^{12-14}$ and all-cause mortality. ${ }^{7}$ Evidence suggests that physical activity can prevent premature death in older adults. $3,8,9,15,16$ Although research has examined carotenoid concentration and physical activity separately in relation to health and survival, less is known about how these factors predict health and survival in concert. ${ }^{17-20}$ In the few studies that have examined this relationship, ${ }^{19}$ the independent, conjoint, and interactive relationships between fruit and vegetable intake and physical activity 
were not investigated. Other studies have examined different outcomes, such as weight gain. ${ }^{20}$

Despite evidence that fruit and vegetable intake and physical activity each improves survival in older adults, more understanding is needed regarding the interrelationship of these factors for predicting vital status. Specifically, do lower fruit and vegetable intake and level of exercise simply reflect poor or declining health, or do they directly and independently contribute to health and survival in older adults? It was hypothesized that a diet high in fruits and vegetables, as reflected by high total serum carotenoid concentrations, combined with high physical activity would predict survival. Furthermore, evidence of additive associations supports a direct contribution of diet and activity to longer survival.

\section{METHODS}

Participants in this study were women aged 70 to 79 from the Women's Health and Aging Study (WHAS) I and II, two complementary, population-based studies designed to evaluate the causes and course of physical disability in older women living in the community. Participants in WHAS I were recruited from an age-stratified random sample of women aged 65 and older selected from Medicare enrollees residing in 12 contiguous ZIP code areas in Baltimore, Maryland. ${ }^{21}$ Women were screened to identify self-reported physical difficulty with tasks in the areas of mobility, upper extremity function, higher-functioning household management, and self-care. WHAS I enrolled the one-third most-disabled women aged 65 and older (those with disability in $\geq 2$ domains). Of the 1,409 women eligible upon screening, 1,002 agreed to participate. Reported health and sociodemographic characteristics did not differ between participants and those who declined. ${ }^{21}$ Standardized questionnaires were administered in participants' homes. A trained registered nurse conducted an examination of each participant using a standardized protocol. Approximately $75 \%$ of the women also consented to phlebotomy. Women who did not participate in the blood drawing had less education and were frailer than those who participated. ${ }^{22}$ Further details of the sampling design and study methods of WHAS I and II have been published elsewhere. ${ }^{21,23}$

Women's Health and Aging Study II was designed as a companion study to WHAS I and included a cohort of women aged 70 to 79 selected to represent the two-thirds least-disabled women living in the community from the same sampling frame used in WHAS I. (Eligible women had no disability or disability in only one domain.) Participants were selected from age-stratified random samples. Eight hundred eighty women were eligible for WHAS II, and 436 consented to participate. Those consenting were more highly educated and reported more diseases than those who refused but did not differ in disability characteristics. ${ }^{7}$ Phlebotomy was performed in $93 \%$ of WHAS II participants following the same protocol as used in WHAS I. Vital status was determined through follow-up interviews with proxies, obituaries, and the National Death Index over a 5 -yr period. The Johns Hopkins University institutional review board approved the study protocol, and written informed consent was obtained from all participants.
For comparability with the WHAS II cohort age range of 70 to 79 , women from WHAS I were included only if they were within this age range, to yield a combined sample of 722 participants with baseline blood samples-303 from WHAS I and 419 from WHAS II. Nine women were excluded because data on important variables were missing, resulting in a final sample of 713 participants. Weights were calculated to adjust for response rates and sampling probability. This pooled sample was used in published studies of undernutrition and frailty, ${ }^{22}$ nutrition and mortality, ${ }^{7}$ and other studies. ${ }^{24,25}$

\section{Study Variables}

Physical activity was assessed using a subset of the Minnesota Leisure Time Physical Activity Questionnaire used in the Cardiovascular Health Study, condensed from the original 18 activities to assess participation in six activities: walking for exercise, doing strenuous household chores (e.g., scrubbing, vacuuming), doing strenuous outdoor chores (e.g., gardening), dancing, bowling, and exercise (e.g., stretching or strengthening activities). ${ }^{26}$ The Minnesota Leisure Time Physical Activity Questionnaire has been found appropriate for epidemiological research with high reliability and variability in adult samples. ${ }^{27-29}$ For each of the six activities, study participants were asked whether they had participated in the activity during the past 2 weeks. Those who responded yes were then asked about the frequency and duration (average time per session) spent in doing the activity in the past 2 weeks. As a continuous measure of level of physical activity, kcal expenditure per $\mathrm{kg}$ of body weight per day (kcal/kg per day) over a 2-week period was calculated by multiplying the activity-specific metabolic equivalent value by the average number of minutes per day spent in the activity and summing across the six activities. ${ }^{30}$ Continuous measures were calculated in log form because of skewness of the distribution. Categorical measures of physical activity were constructed from the National Health Interview Survey criterion of sedentary $(<1.5 \mathrm{kcal} / \mathrm{kg}$ per day), moderately active (1.5-2.9 kcal/kg per day), and most active $\left(\geq 3.0 \mathrm{kcal} / \mathrm{kg}\right.$ per day). ${ }^{31}$

Total carotenoids $(\mu \mathrm{mol} / \mathrm{L})$ were calculated at baseline as the sum of alpha-carotene, beta-carotene, beta-cryptoxanthin, lutein plus zeaxanthin, and lycopene. Carotenoid levels were measured from nonfasting blood samples obtained using venipuncture. Processing, aliquoting, and freezing processes were performed following a standardized protocol at the Core Genetics Laboratory of The Johns Hopkins University School of Medicine. Blood samples were analyzed for carotenoids using high-performance liquid chromatography. ${ }^{32}$ Categorical measures of total carotenoids consisted of tertiles from the present sample (lowest, $<1.350$; middle, 1.350-2.059; highest, $\geq 2.060$ $\mathrm{umol} / \mathrm{L})$.

Sociodemographic covariates in the analysis were age, level of education ( $\geq 12$ vs $<12$ years), and race or ethnicity (white vs not white) at baseline.

To evaluate physical activity and total carotenoids conjointly, groups were constructed based on National Health Interview Survey categories of physical activity and tertiles of total serum carotenoids at baseline. The most 
active women and those in the highest tertile of total serum carotenoids were assigned to Group 1, those in the highest tertile of carotenoids and sedentary at baseline were assigned to Group 2, those who were the most active and had the lowest tertile of carotenoids at baseline were assigned to Group 3, those who were sedentary and in the lowest tertile of total serum carotenoids were assigned to Group 4, and those who were moderately active or were in the middle tertile of total serum carotenoids were assigned to Group 5.

Additional health and chronic disease covariates were included in supplementary analyses. These covariates were selected because of their strong mechanistic associations with serum carotenoid levels, physical activity, or mortality. Health-related covariates included body mass index $\left(\mathrm{kg} / \mathrm{m}^{2}\right)$, health behaviors, and chronic disease status at baseline. Whether the participant smoked or drank was assessed according to self-report. Body mass index (BMI) was categorized as underweight $\left(<18.5 \mathrm{~kg} / \mathrm{m}^{2}\right)$, normal $\left(18.5-24.9 \mathrm{~kg} / \mathrm{m}^{2}\right)$, overweight $\left(\geq 25.0-29.9 \mathrm{~kg} / \mathrm{m}^{2}\right)$, or obese $\left(\geq 30.0 \mathrm{~kg} / \mathrm{m}^{2}\right)$ according to World Health Organization criteria. ${ }^{33}$ Trained physicians adjudicated the presence or absence of chronic diseases using abstracted medical records and following standardized algorithms. ${ }^{21}$ Cardiovascular disease was defined as coronary heart disease, congestive heart failure, or peripheral artery disease. Chronic kidney disease was defined as estimated glomerular filtration rate $<60 \mathrm{~mL} / \mathrm{min}$ per $1.73 \mathrm{~m}^{2}$ using the four-variable Modification of Diet in Renal Disease Study equation. $^{34}$

\section{Statistical Analysis}

Means and percentages were used to describe the characteristics of the study population. Groups were compared using $t$-tests for continuous variables and chi-square tests for categorical variables. Cox proportional hazards models were used to examine the relationship between physical activity and fruit and vegetable intake for women in each group over 5 years of follow-up. The proportional hazards assumption was tested using the sphtest command in Stata (StataCorp., College Station, TX), which is a test of a nonzero slope in a generalized linear regression of the scaled Schoenfeld residuals on time. The test for the proportional hazards assumption test was not statistically significant, indicating no evidence of violation of the assumption. Hazard ratios (HRs) and confidence intervals (CIs) are presented for categorical measures. To improve interpretation, the HRs have been transformed using sample standard deviation (SD) units in the multivariate model for continuous measures (log of continuous physical activity and carotenoids). Stata SE (StataCorp) was used for data management and analysis. $P<.05$ was considered significant.

\section{RESULTS}

Of the 713 participants, 377 were sedentary, 147 were moderately active, and 189 were in the most active group at baseline. Demographic characteristics are shown according to physical activity and total serum carotenoid category in Table 1. BMI and age were inversely associated with physical activity level; women who reported alcohol consumption engaged in higher levels of physical activity. Women who smoked had lower levels of serum carotenoids, whereas women who drank alcohol had higher levels.

Over 5 years of follow-up, $82(11.5 \%)$ participants died. Demographic characteristics are shown according to survival status in Table 2 . Women who died were more likely to be nonwhite. Women with lower continuous measures of total serum carotenoid concentrations at baseline and lower continuous measures of physical activity at baseline had significantly greater mortality.

The six multivariate Cox proportional hazards models for 5-year all-cause mortality are presented in Table 3. HRs are provided for models that evaluate categorical measures of physical activity, as indicated in Table 3. Model 1 included a continuous measure of physical activity and demographic covariates. A 1-standard deviation increase in the log of physical activity predicted a $48 \%$ lower risk of mortality over 5 years $(\mathrm{HR}=0.52,95 \%$ $\mathrm{CI}=0.41-0.66, P<.001)$. As shown in Model 2 , the most physically active women were more likely than sedentary women to survive $(\mathrm{HR}=0.28$, 95\% CI $=0.13$ $0.59, P<.001)$; in other words, being most physically active resulted in a $72 \%$ lower 5 -year mortality risk than being sedentary.

Table 1. Characteristics of the Study Population at Baseline (Weighted)

\begin{tabular}{|c|c|c|c|c|c|c|c|c|}
\hline \multirow[b]{2}{*}{ Characteristic } & \multicolumn{4}{|c|}{ Physical Activity } & \multicolumn{4}{|c|}{ Carotenoids } \\
\hline & $\begin{array}{l}\text { Sedentary } \\
(\mathrm{n}=377)\end{array}$ & $\begin{array}{l}\text { Moderately Active } \\
\quad(n=147)\end{array}$ & $\begin{array}{c}\text { Most Active } \\
(n=189)\end{array}$ & $\begin{array}{c}P \text { - } \\
\text { value }\end{array}$ & $\begin{array}{c}\text { Low } \\
(n=239)\end{array}$ & $\begin{array}{l}\text { Medium } \\
(n=239)\end{array}$ & $\begin{array}{c}\text { High } \\
(n=235)\end{array}$ & $\begin{array}{c}P \text { - } \\
\text { value }\end{array}$ \\
\hline Age, mean $\pm S D$ & $74.2 \pm 2.8$ & $73.6 \pm 2.9$ & $74.0 \pm 2.9$ & .02 & $73.9 \pm 2.8$ & $74.1 \pm 2.9$ & $74.1 \pm 2.9$ & .46 \\
\hline Education $<12$ years, $\%$ & 67.9 & 67.4 & 68.8 & .9 & 64.9 & 69.0 & 70.2 & .33 \\
\hline White, \% & 72.7 & 80.3 & 83.6 & .1 & 76.2 & 80.8 & 74.5 & .23 \\
\hline $\begin{array}{l}\text { Body mass index, } \mathrm{kg} / \mathrm{m}^{2} \text {, } \\
\text { mean } \pm \mathrm{SD}\end{array}$ & $28.6 \pm 6.5$ & $26.7 \pm 5.1$ & $26.7 \pm 5.4$ & $<.001$ & $28.7 \pm 6.4$ & $28.0 \pm 6.1$ & $26.4 \pm 5.3$ & .23 \\
\hline Diabetes mellitus, \% & 14.6 & 12.9 & 7.4 & .60 & 15.9 & 10.0 & 11.1 & .60 \\
\hline Cardiovascular disease, \% & 31.0 & 23.8 & 15.3 & .09 & S & 23.9 & 21.7 & .09 \\
\hline Renal disease, \% & 49.4 & 57.6 & 50.8 & .11 & 55.3 & 50.7 & 48.7 & .11 \\
\hline
\end{tabular}

$\mathrm{SD}=$ standard deviation. 
Table 2. Characteristics of the Study Population According to Survival During Follow-Up

\begin{tabular}{lllc}
\hline \multicolumn{1}{c}{ Characteristic } & $\begin{array}{c}\text { Survived } \\
(\mathbf{n = 6 3 1 )}\end{array}$ & $\begin{array}{c}\text { Died } \\
(\mathbf{n}=\mathbf{8 2})\end{array}$ & $\begin{array}{c}\boldsymbol{P} \text { - } \\
\text { Value }\end{array}$ \\
\hline Age, mean $\pm \mathrm{SD}$ & $74.0 \pm 2.8$ & $74.4 \pm 2.8$ & .14 \\
Education $<12$ years, \% & 69.1 & 59.8 & .09 \\
White, \% & 78.3 & 68.3 & .04 \\
Current smoker, \% & 10.6 & 23.2 & .001 \\
Uses alcohol, \% & 26.8 & 17.1 & .06 \\
Body mass index, kg/m ${ }^{2}$, & $27.8 \pm 5.8$ & $26.9 \pm 7.7$ & .24 \\
mean \pm SD & & & \\
Diabetes mellitus, \% & 10.0 & 30.5 & $<.001$ \\
Cardiovascular disease, \% & 22.8 & 45.1 & $<.001$ \\
Renal disease, \% & 51.9 & 48.7 & .60 \\
Total serum carotenoids, $\mu \mathrm{mol} / \mathrm{L}$, & $1.9 \pm 0.9$ & $1.7 \pm 0.7$ & .03 \\
mean \pm SD & & & \\
Total physical activity $(\mathrm{kcal} / \mathrm{kg}$ & $2.8 \pm 3.3$ & $1.3 \pm 2.2$ & $<.001$ \\
per day expenditure over a & & & \\
2-week period), mean \pm SD & & & \\
\hline
\end{tabular}

$\mathrm{SD}=$ standard deviation

Fruit and vegetable intake also predicted mortality. As shown in Model 3, one 1-standard deviation increase in total serum carotenoids predicted a $33 \%$ lower 5-year mortality risk $\quad(\mathrm{HR}=0.67, \quad 95 \% \quad \mathrm{CI}=0.51-0.89$, $P=001$ ). When tested categorically (Model 4), women in the highest carotenoid group had $50 \%$ longer survival than women in the lowest group $(\mathrm{HR}=0.50,95 \% \mathrm{CI}=0.27$ $0.91, P=.003$ ).

When examined together (Model 5), physical activity and total serum carotenoids as continuous measures predicted better survival. A 1-standard deviation increase in the $\log$ of physical activity predicted $58 \%$ lower mortality $(\mathrm{HR}=0.42,95 \% \mathrm{CI}=0.42-0.68, P<.001)$, and a 1 standard deviation increase in total carotenoids was associated with $24 \%$ lower 5 -year mortality $(\mathrm{HR}=0.76,95 \%$ $\mathrm{CI}=0.59-0.98, P=004)$. Categorical measures of physical activity and carotenoids also predicted survival; as shown in Model 6, women in the most-active group $(\mathrm{HR}=0.29,95 \% \mathrm{CI}=0.14-0.62, P<.001)$ and women in the highest tertile of total carotenoids ( $\mathrm{HR}=0.54,95 \%$ $\mathrm{CI}=0.29-0.99, P=.047)$ were the most likely to survive the 5-year follow up period. Women in the most-active group at baseline had $71 \%$ lower 5-year mortality, and women in the highest carotenoid group at baseline had $46 \%$ lower 5 -year mortality than the least-active and lowest carotenoid groups, respectively. There was no interaction between the continuous measures of physical activity and total carotenoids $(P=.10)$ or any combination of the categorical measures $(P>.61$ for all).

Overall, associations weakened when the models included health- and disease-related covariates. Categorical and continuous measures of carotenoids were no longer significant predictors of 5 -year mortality after adjusting for additional confounders.

The multivariate Cox proportional hazards models that examined physical activity and total carotenoids as conjoint predictors of 5 -year all-cause mortality are presented in Table 4, with women in the highest tertile of carotenoids who were also the most physically active as the reference group. Sedentary women in the highest

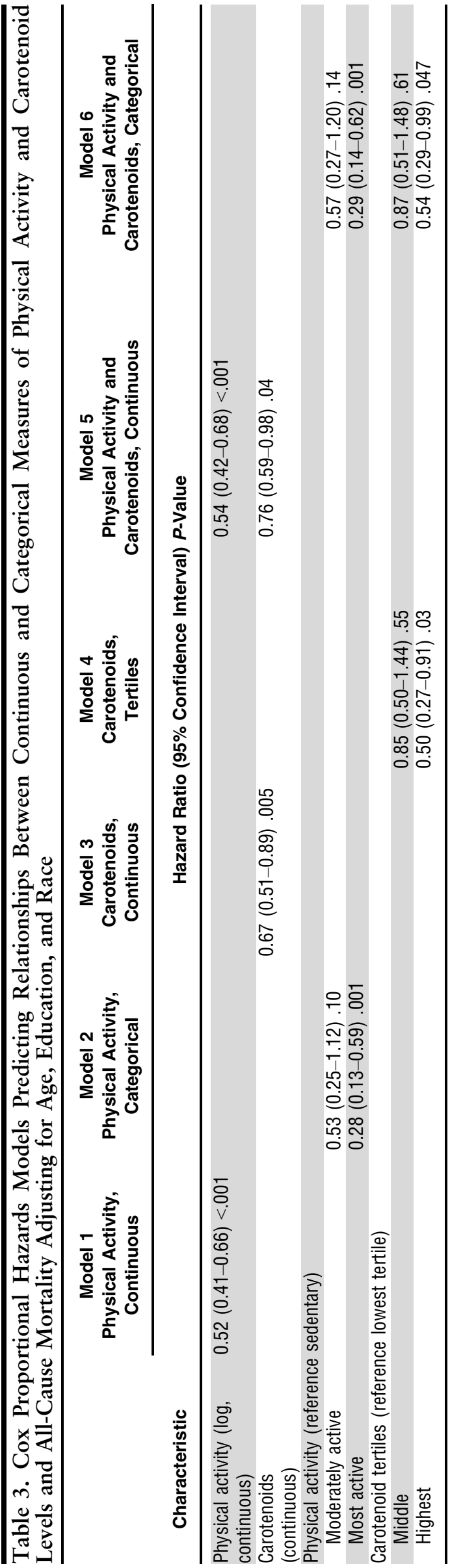


carotenoid tertile no longer experienced significantly greater 5-year mortality than this group. Women who were the most physically active but who were in the lowest carotenoid tertile had significantly greater mortality as well $(\mathrm{HR}=4.83,95 \% \quad \mathrm{CI}=1.34-17.43, \quad P=.02)$. Women who were the least physically active and who were in the lowest tertile of total carotenoids had the highest mortality $(\mathrm{HR}=7.86,95 \% \mathrm{CI}=2.27-27.19, P<.001)$, controlling for age, education, and race and ethnicity. When healthand disease-related covariates were included, the HRs associated with high physical activity plus low carotenoids and low physical activity plus low carotenoids diminished but remained statistically significant.

If there was a synergistic association between physical activity and carotenoids, the product of the HRs for Groups 2 (high carotenoid and low activity) and 3 (low carotenoid and high activity) would be expected to be substantially smaller than the HR for Group 4 (low carotenoid and low activity) under a multiplicative model such as the Cox model. The product of the HRs for Groups 2 and 3 was 10.67, which is larger than the HR for Group 4, which was 7.86, indicating no evidence of interaction between physical activity and carotenoids in this study.

\section{DISCUSSION}

The present study found that physical activity and total serum carotenoids are strong and independent predictors of survival in older women living in the community. This offers preliminary support for the hypothesis that a diet high in fruit and vegetables, as reflected by high total serum carotenoid concentrations, combined with high physical activity would each demonstrate a protective association with 5-year mortality independent of one another. Therefore, exercise and nutrition should both be analyzed when assessing the health and projected life span of older women. Programs and policies to promote longevity should include interventions to improve nutrition and physical activity in older adults.

Table 4. Cox Proportional Hazards Model Demonstrating Conjoint Relationships Between Physical Activity, Carotenoid Levels, and All-Cause Mortality After Adjusting for Age, Education, and Race

\begin{tabular}{lcccc}
\hline \multicolumn{1}{c}{ Group } & $\begin{array}{c}\text { Sample } \\
\text { Size, }\end{array}$ & $\begin{array}{c}\text { Deaths, } \\
\mathbf{n}\end{array}$ & $\begin{array}{c}\text { Hazard Ratio } \\
\text { (95\% Confidence } \\
\text { Interval) }\end{array}$ & $\begin{array}{c}\boldsymbol{P} \text { - } \\
\text { Value }\end{array}$ \\
\hline $\begin{array}{l}1 \text { (reference: } \\
\text { high carotenoid, } \\
\text { high physical }\end{array}$ & 72 & 3 & 1.0 & \\
$\begin{array}{l}\text { activity) } \\
2 \text { (high } \\
\text { carotenoid, low } \\
\text { physical activity) }\end{array}$ & 57 & 4 & $2.21(0.46-10.53)$ & .32 \\
$\begin{array}{l}3 \text { (low } \\
\text { carotenoid, high } \\
\text { physical activity) }\end{array}$ & 105 & 16 & $4.83(1.34-17.43)$ & .02 \\
$\begin{array}{l}4 \text { (low } \\
\text { carotenoid, low } \\
\text { physical activity) }\end{array}$ & 145 & 25 & $7.86(2.27-27.19)$ & $<.001$ \\
5 (other) & 334 & 34 & $4.21(1.23-14.35)$ & .02 \\
\hline
\end{tabular}

Through the examination of physical activity and carotenoid tertiles, the study also found that conjoint relationships of physical activity and carotenoid levels predicted 5-year mortality. The significant conjoint effects suggest that different combinations of physical activity and diet levels are predictive of longevity, offering support for the hypothesis of a joint association between diet and activity and survival. Participants with low levels of both attributes were nearly eight times as likely to experience mortality during the follow-up period as those with high levels of both. Participants with high carotenoid values and low exercise were more than twice as likely to die, whereas those with low carotenoid values and high levels of exercise were nearly four times as likely. These conjoint analyses suggest that the combination of physical activity and diet predicts better survival and that a low level on one measure (particularly carotenoids) is also associated with mortality risk. In practice, this finding suggests that high levels of both attributes are strong predictors of better survival, whereas low levels of both greatly increase the risk of death. Increasing healthpromoting habits in one aspect (e.g., physical activity) does not compensate for inadequate attention to another (e.g., diet quality). This further suggests that interventions should be multifaceted, promoting high levels of fruit and vegetable intake and high levels of physical activity in older women.

Although fruit and vegetable intake and physical activity independently and conjointly predict earlier mortality, they do not have an interactive protective effect. Significant interactive effects were found in a prior analysis, ${ }^{20}$ but there was no evidence suggesting a significant or underlying interaction in this study.

Few studies have examined the relationship between diet, exercise, and mortality risk. ${ }^{19}$ To the knowledge of the authors of the current study, this is the first study to show that the combination of a healthy diet and greater physical activity predict greater survival and that the combination of both positive factors confers lower mortality risk than either factor alone.

Older adults are at risk of undernutrition, ${ }^{35}$ and the majority of older adults do not reach the recommended levels of daily fruit and vegetable intake. According to a Centers for Disease Control and Prevention surveillance study, fewer than one-third of adults aged 65 and older eat recommended daily values of fruits and vegetables. ${ }^{36}$ Given the strong protective effect of diet-independent of physical activity - a multidisciplinary approach toward improving the diet of older adults should be taken in policy and in practice. Although the present study is limited in that dietary intake was not measured, higher carotenoid concentrations are known to be strongly associated with higher fruit and vegetable intake. ${ }^{37}$

Greater physical activity was a significant predictor of better survival, but previous research has found that even small amounts of physical activity are protective against health decline in elderly adults. ${ }^{38}$ Unfortunately, $35.1 \%$ of adults aged 65 to 74 and $46 \%$ of those aged 75 and older do not engage in any leisure-time physical activity. ${ }^{36}$ Interventions that encourage physical activity should provide opportunities for mobility-limited older adults to participate. 
There are several potential mechanisms by which diet and physical activity could conjointly promote longevity. Carotenoids provide antioxidants that can protect against oxidative stress, inflammation, and cardiovascular disease. $^{39-41}$ The examination of protective effects of certain carotenoids can also reveal protective pathways. For example, beta-carotene and alpha-carotene levels-which could decrease oxidized lipids and decrease activation of inflammatory pathways - protect against coronary artery disease in adult women. ${ }^{42-46}$ Physical activity contributes to muscle strength and mobility, which improve longevity in older adults. ${ }^{47}$ Physical activity also protects against adiposity. ${ }^{16}$ Furthermore, combined healthful physical activity and dietary practices prevent complications and mortality associated with chronic illnesses, such as diabetes mellitus and cardiovascular disease, which independently predicted mortality in this analysis. Interventions to improve the health of older adults have traditionally focused on improving nutritional status or increasing physical activity. This study provides motivation for a two-pronged approach for better longevity in this population.

This study has several limitations. First, the sample was limited to women aged 70 to 79 , although it offers evidence from the full spectrum of health and functional status of community-dwelling women in this age range. The extent to which these findings can be generalized to younger or older male populations is not known and warrants further study. Another limitation concerns possible residual confounding in the multivariate models due to measurement error and incomplete characterization of variables included in the models, given that only one set of measurements was used to determine baseline physical activity and carotenoid levels.

The HRs associated with physical activity and carotenoid levels diminish after controlling for health and chronic disease covariates. It is likely that this is because of overadjustment of the model. For example, high BMI is a cause and a consequence of low physical activity and is associated with earlier mortality, which could lead to confounding. Furthermore, health behaviors and chronic diseases examined as covariates in supplementary analyses could be mediators - or direct correlates of mediators - in the causal pathway of the effects of physical activity and carotenoids on longevity. By controlling for these comorbidities, it is possible that much of the estimated protective effects of fruit and vegetable intake and physical activity on survival is being removed.

\section{CONCLUSION}

Although the present study provides evidence that physical activity and fruit and vegetable intake are independently and jointly related to longevity, further work is required to validate and extend these findings in other populations so that appropriate groups can be targeted for interventions that incorporate diet and physical activity. The implications of this work are that interventions should combine improvements in diet and physical activity - rather than examine changes in isolation - to improve survival in older populations.

\section{ACKNOWLEDGMENTS}

Conflict of Interest: The editor in chief has reviewed the conflict of interest checklist provided by the authors and has determined that the authors have no financial or any other kind of personal conflicts with this paper.

Funding was provided by the National Institute on Aging.

Author Contributions: Emily N. and Richard S.: conceived of the study hypothesis. Qian-Li X. and Emily N.: designed the overall analysis plan. Jing T., Kai S., Anne C., Eleanor S., Luigi F., and Linda F.: contributed to data interpretation and preparation of the manuscript.

Sponsor's Role: The sponsor did not have a role in the design, methods, or other aspects of this manuscript.

\section{REFERENCES}

1. Crimmins EM, Saito Y. Change in the prevalence of diseases among older Americans: 1984-1994. Demographic Res 2000;3:1-20.

2. Rosén M, Haglund B. From healthy survivors to sick survivors: Implications for the 21st century. Scand J Public Health 2005;33:151-155.

3. Fried LP, Kronmal RA, Newman AB, et al. Risk factors for 5-year mortality in older adults: The Cardiovascular Health Study. JAMA 1998;279:585592.

4. Mokdad AH, Marks JS, Stroup DF, et al. Actual causes of death in the United States, 2000. JAMA 2004;291:1238-1245.

5. De Waart FG, Shouten EG, Stalenhoef AFH, et al. Serum carotenoids, $\alpha-$ tocopherol and mortality risk in a prospective study among Dutch elderly. Int J Epidemiol 2001;30:136-141.

6. Lauretani F, Semba RD, Dayhoff-Brannigan M, et al. Low total plasma carotenoids are independent predictors of mortality among older persons: The InCHIANTI Study. Eur J Nutr 2008;47:335-340.

7. Ray AL, Semba RD, Walston J, et al. Low serum selenium and total carotenoids predict mortality among older women living in the community: The Women's Health and Aging Studies. J Nutrition 2006;136:172-176.

8. Anderson LB, Schnohr P, Schroll M, et al. All-cause mortality associated with physical activity during leisure time, work, sports, and cycling to work. Arch Intern Med 2000;160:1621-1628.

9. Kushi LH, Fee RM, Folsom AR, et al. Physical activity and mortality in postmenopausal women. JAMA 1997;277:1287-1292.

10. Semba RD, Varadhan R, Bartali B, et al. Low serum carotenoids and development of severe walking disability among older women living in the community: The Women's Health and Aging Study I. Age Ageing 2007;36:62-67.

11. Morris DL, Kritchevsky SB, Davis CE. Serum carotenoids and coronary heart disease. JAMA 1994;272:1439-1441.

12. Gaziano JM, Manson JE, Branch LG, et al. A prospective study of carotenoids in fruits and vegetables and decreased cardiovascular mortality in the elderly. Ann Epidemiol 1995;5:255-260.

13. Ito Y, Yakai K, Suzuki K, et al. Serum carotenoids and mortality from lung cancer: A case-control study nested in the Japan Collaborative Cohort (JACC) Study. Cancer Sci 2003;94:57-63.

14. Ito $\mathrm{Y}$, Kurata M, Hioki R, et al. Cancer mortality and serum levels of carotenoids, retinol, and tocopherol: A population-based follow-up study of inhabitants of a rural area of Japan. Asian Pacific J Cancer Prev 2005;6: 10-15.

15. Katzmarzyk PT, Janssen I, Ardern CI. Physical inactivity, excess adiposity and premature mortality. Obes Rev 2003;4:257-290.

16. Hu FB, Willett WC, Li T, et al. Adiposity as compared with physical activity in predicting mortality among women. N Engl J Med 2004;351:26942703.

17. World Health Organization, Food and Agriculture Organization of the United Nations. Diet, Nutrition and the Prevention of Chronic Diseases. WHO Technical Report Series 916. 2003. Available at: http://www.who. int/hpr/NPH/docs/who_fao_expert_report.pdf Accessed March 1, 2011.

18. Drewnowski A, Evans WJ. Nutrition, physical activity and quality of life in older adults. J Gerontol A Biol Sci Med Sci 2001A;56A:89-94.

19. Knoops K, de Groot L, Kromhout D, et al. Mediterranean diet, lifestyle factors, and 10-year mortality in elderly European men and women. JAMA 2004;292:1433-1439. 
20. Mozaffarian D, Hao T, Rimm EB, et al. Changes in diet and lifestyle and long-term weight gain in women and men. N Engl J Med 2011;364:23922404.

21. Guralnik JM, Fried LP, Simonsick EM et al. The Women's Health and Aging Study: Health and Social Characteristics of Older Women with Disability (NIH Publication No. 95-4009). Bethesda, MD: National Institute on Aging, 1995.

22. Michelon E, Blaum C, Semba RD, et al. Vitamin and carotenoid status in older women: associations with The frailty syndrome. J Gerontol A Biol Sci Med Sci 2006;61A:600-607.

23. Fried LP, Bandeen-Roche K, Kasper JD, et al. Association of comorbidity with disability in older women: The Women's Health and Aging Study. J Clin Epidemiol 1999;52:27-37.

24. Blaum CS, Xue QL, Michelon E, et al. The association between obesity and the frailty syndrome in older women: The Women's Health and Aging Studies. J Am Geriatr Soc 2005;53:927-934.

25. Cappola AR, Bandeen-Roche K, Wand GS, et al. Association of IGF-1 levels with muscle strength and mobility in older women. J Clin Endocrinol Metab 2001;86:4139-4146.

26. Taylor HL, Jacobs DR Jr, Schucker B, et al. A questionnaire for assessment of leisure time physical activities. J Chronic Dis 1978;31:741-755.

27. Folsom AR, Jacobs DR, Caspersen CJ, et al. Test-retest reliability of the Minnesota Leisure Time Physical Activity Questionnaire. J Chron Dis 1986;39:505-511.

28. Elosua R, Garcia M, Aguilar A, et al. Validation of the Minnesota Leisure Time Physical Activity Questionnaire in Spanish women. Med Sci Sports Exerc 2000;32:1431-1437.

29. Eckel SP, Bandeen-Roche K, Chaves PH, et al. Surrogate screening model for the low physical activity criterion for frailty. Aging Clin Exp Res 2011;23:209-216.

30. Ainsworth BE, Haskell WL, Leon AS, et al. Compendium of physical activities: Classification of energy costs of human physical activities. Med Sci Sports Exerc 1993;25:71-80.

31. Healthy People 2000 (DHHS Publication No. (PHS) 91-50212). Washington, DC: U.S. Government Printing Office, 1990.

32. Semba RD, Blaum C, Guralnik JM, et al. Low carotenoid and vitamin E status are associated with indicators of sarcopenia among older women living in the community. Aging-Clin Exp Res 2003;15:482-487.

33. World Health Organization. Obesity: Preventing and Managing the Global Epidemic. Report on a WHO Consultation on Obesity, Geneva, June 3-5, 1997, WHO/NUT/NCD/98.1, Geneva, Switzerland: World Health Organization, 1998.
34. Levey AS, Bosch JP, Lewis JB, et al. A more accurate method to estimate glomerular filtration rate from serum creatinine: A new prediction equation. Ann Intern Med 1999;130:461-470.

35. Foote JA, Giuliano AR, Harris RB. Older adults need guidance to meet nutritional recommendations. J Am Coll Nutr 2000;19:628-640.

36. Centers for Disease Control Prevention. Surveillance for five health risks among older adults, United States, 1993-1997. MMWR Surveill Studies 1999;48:89-130.

37. Food Nutrition Board, Institute of Medicine. Dietary Reference Intakes for Vitamin C, Vitamin E, Selenium, and Carotenoids. Washington, DC: National Academy Press, 2000.

38. Simonsick EM, Guralnik JM, Volpato S, et al. Just get out the door! Importance of walking outside the home for maintaining mobility: Findings from the Women's Health and Aging Study. J Am Geriatr Soc 2005;53:198 -203 .

39. Chrysohoou C, Panagiotakos DB, Pitsavos C, et al. Adherence to the Mediterranean diet attenuates inflammation and coagulation process in healthy adults: The Attica Study. J Am Coll Cardiol 2004;44:152-158.

40. Esposito K, Marfella R, Ciotola M, et al. Effect of a Mediterranean-style diet on endothelial dysfunction and markers of vascular inflammation in the metabolic syndrome: A randomized trial. JAMA 2004;292:1440 1446.

41. Lopes HF, Martin KL, Nashar K, et al. DASH diet lowers blood pressure and lipid-induced oxidative stress in obesity. Hypertension 2003;41:422430 .

42. Osganian SK, Stampfer MJ, Rimm E, et al. Dietary carotenoids and risk of coronary artery disease in women. Am J Clin Nutr 2003;77:1390 1399.

43. Kontush A, Spranger T, Reich A, et al. Lipophilic antioxidants in blood plasma as markers of atherosclerosis: The role of alpha-carotene and gamma-tocopherol. Atherosclerosis 1999;144:117-122.

44. Kontush A, Weber W, Beisiegel U. Alpha- and beta-carotenes in low density lipoprotein are the preferred target for nitric oxide-induced oxidation. Atherosclerosis 2000;148:87-93.

45. Blackwell TS, Christman JW. The role of nuclear factor- $\beta$ in cytokine gene regulation. Am J Respir Cell Mol Biol 1997;17:3-9.

46. Kunsch C, Medford RM. Oxidative stress as a regulator of gene expression in the vasculature. Circ Res 1999;85:753-766.

47. Rantanen T, Guralnik JM, Sakari-Rantala R, et al. Disability, physical activity, and muscle strength in older women: The Women's Health and Aging Study. Arch Phys Med Rehab 1999;80:130-135. 